\title{
Emerging roles of E3 ubiquitin ligases in autophagy
}

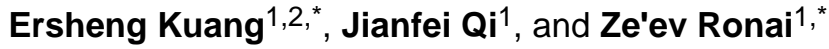 \\ ${ }^{1}$ Signal Transduction Program, Sanford-Burnham Medical Research Institute, La Jolla, CA 92037, \\ USA \\ ${ }^{2}$ Institute of Human Virology, Zhongshan School of Medicine, Sun Yat-sen University and Key \\ Laboratory of Tropical Disease Control, Ministry of Education, Guangzhou 510080, China
}

\begin{abstract}
Autophagy is an evolutionarily conserved intracellular catabolic process that delivers cytoplasmic components to lysosomes for degradation and recycling. Although originally considered to be a non-selective pathway, it is now recognized that autophagy is involved in selective processes, including the turnover of organelles, removal of protein aggregates, and elimination of intracellular pathogens. This specificity implies that cargo recognition and processing by the autophagy machinery are tightly regulated processes. In support of this, various forms of posttranslational modification have been implicated in the regulation of autophagy, one of which is the ubiquitin-proteasome system. Here, we review the current understanding of the role of ubiquitylation in the control of autophagy.
\end{abstract}

\section{Keywords}

Ubiquitin ligase; Proteasome; Autophagy; Parkin; RNF5

\section{Autophagy}

Autophagy is a major catabolic process that delivers cytoplasmic components to lysosomes for degradation and recycling. Several forms of autophagy have been described: macroautophagy, microautophagy, and chaperone-mediated autophagy. In microautophagy, cytoplasmic components are directly engulfed by lysosomes, and in chaperone-mediated autophagy, proteins undergo receptor-mediated translocation into lysosomes. Macroautophagy (hereafter referred to as autophagy) is the most well characterized form of autophagy. Autophagy is initiated by the formation of phagophores (cup-shaped membrane structures), which engulf components of the cytoplasm. The phagophore elongates and seals to form a double membrane vesicle: the autophagosome. The autophagosome then fuses with a lysosome, which results in degradation of the cargo [1, 2]. Autophagy has long been considered to be a non-selective response to starvation, in which bulk cytoplasmic components are recycled to provide nutrients and maintain cellular homeostasis. However, it is now well established that autophagy is a highly selective process, and can be further categorized according to the specific substrates targeted for removal; namely, aggrephagy (removal of aggregated proteins), pexophagy (peroxisomes), mitophagy (mitochondria),

\footnotetext{
(C) 2013 Elsevier Ltd. All rights reserved.

"Correspondence: kuangersh@mail.sysu.edu.cn, ronai@sanfordburnham.org.
}

Publisher's Disclaimer: This is a PDF file of an unedited manuscript that has been accepted for publication. As a service to our customers we are providing this early version of the manuscript. The manuscript will undergo copyediting, typesetting, and review of the resulting proof before it is published in its final citable form. Please note that during the production process errors may be discovered which could affect the content, and all legal disclaimers that apply to the journal pertain. 
xenophagy (viruses and bacteria), reticulophagy (endoplasmic reticulum; ER), and ribophagy (ribosomes). This highly selective and evolutionarily conserved (from yeast to mammals) process thus contributes to the maintenance of key physiological processes, including metabolism, development, programmed cell death, and aging [3-6]. Autophagy also plays an important role in the cellular response to stress; for example, as a defense against pathogen infection [7-10]. Consistent with the critical role of autophagy in normal cell function, deregulation of the process has been implicated in a number of pathological conditions, including neurodegenerative disorders and cancer, as well as in the cellular response to chemotherapeutic agents [11-17]. Therefore, a thorough understanding of the molecular mechanisms by which autophagy is regulated might lead to new treatment options for a number of diseases.

\section{Molecular mechanisms of the ubiquitin-proteasome system and autophagy}

Ubiquitylation plays a central role in controlling the protein stability, activity, localization and protein-protein interactions. The ubiquitylation machinery modifies proteins by selectively conjugating them with ubiquitin chains of different topologies [18, 19]. Ubiquitin is a highly conserved 76-amino acid protein that is conjugated to lysine residues of target proteins by the concerted action of three classes of ubiquitin ligases: E1 (ubiquitin-activating enzymes), E2 (ubiquitin-conjugating enzymes), and E3 (substrate-specific ubiquitin ligases) [20]. In mammals there are more than 500 E3 ligases, which recognize distinct substrate(s), thereby establishing specificity in ubiquitylation [21], as well as 30 E2 conjugating enzymes and 2 E1 activating enzymes. E2 conjugating enzymes, in concert with the E3 and the substrate, determine the type of ubiquitin chain formed (i.e. K48, K63, K11 topologies). Accordingly, substrate ubiquitylation can result in multiple distinct ubiquitin chain configurations [21-23] that are associated with different outcomes. For example, K48-linked polyubiquitylation is primarily implicated in protein degradation; K27- and K29-linked ubiquitylation has been associated with lysosomal degradation [24, 25]; K11-linked polyubiquitylation is involved in cell cycle control and ER-associated degradation (ERAD) of misfolded proteins; and K63-linked and linear ubiquitin chains have been implicated in regulating signal transduction pathways, including the IKK-NF-KB pathway. In addition, mono-ubiquitylation of substrates has been associated with endocytosis and altered subcellular localization [21,22]. The ubiquitin machinery also includes a number of deubiquitylating enzymes (DUBs) that reverse the signal for protein polyubiquitylation by trimming ubiquitin chains.

Whereas the ubiquitin-proteasome system provides a mechanism to control the removal of single or small complex molecules, autophagy represents a complementary process for the removal of macromolecules and organelles. The molecular machinery of autophagy was first elucidated by genetic studies in yeast, which identified $\sim 35$ autophagy-related genes $(A T G)$ that encode components of several functional units, including the ULK (ATG1-Unc51-like kinase) complex, the Beclin 1-interacting complex, and the conjugation machinery (ATG12 and ATG8) [26-28]. The order of recruitment of these proteins to membranes and the contribution of each to the distinct steps in the autophagy process have been elucidated.

Amino acid starvation induces autophagy through inhibition of the mammalian target of rapamycin (mTOR) as part of mTOR Complex 1 (mTORC1). Under normal growth conditions mTORC1 negatively regulates the ULK1 complex, thereby inhibiting the activation of autophagy. When the pool of amino acids is depleted mTORC1 activity is attenuated, leading to autophagy initiation. Another important regulator of autophagy is the Beclin 1-interacting complex, which generates phosphatidylinositol-3-phosphate (PI3P) via Vps34 (yeast) or Class III PI3K (mammals) to promote the nucleation of autophagosomal membranes [1,2]. 
Two ubiquitin-like conjugation systems have been identified as part of the core autophagy machinery essential for elongation of the pre-autophagosomal membrane in mammals (see Figure 1). The first is the ATG12-ATG5-ATG16L1 conjugation system. The ubiquitin-like protein ATG12 is conjugated to ATG5 by ATG7 (E1-like) and ATG10 (E2-like) enzymes. The resulting ATG5-ATG12 interacts with ATG16L1 to form a dimeric complex at the membrane that is essential for elongation of the pre-autophagosomal membrane [29-31]. A second conjugation system involves Atg8-like proteins (yeast Atg8 has number of human homologs including LC3, GABARAP, and GATE-16, of which LC3 is the best characterized). LC3 is cleaved by the cysteine protease ATG4 to generate LC3-I, exposing a $\mathrm{C}$-terminal glycine residue that serves as a lipid conjugation site. Conjugation of phosphatidylethanolamine (PE) with LC3-I is mediated by amide linkage between the Cterminal glycine of LC3-I and an amino group of PE [32], resulting in the formation of LC3II. The ubiquitin-like LC3 is covalently conjugated to PE by the sequential action of ATG7 (E1-like) and ATG3 (E2-like) [30,31,33-35]. In mammals, the conversion of LC3-I to LC3II (LC3-PE) is a key regulatory step in the elongation of autophagosomal membrane. In addition, LC3 is also involved in cargo selection through its LC3-interacting region (LIR) [32]. Notably, the protease ATG4 not only mediates the proteolytic processing of the fulllength isoform of LC3 (pro-LC3), but also is capable of deconjugating the lipidated LC3-PE (LC3-II) to recycle LC3.[33-36] This reversible ubiquitin-like modification plays an important role in the autophagic process.

\section{Role of ubiquitylation in the regulation of autophagy}

Given the fundamental role of autophagy in normal cellular homeostasis, it is not surprising that it is tightly controlled at multiple levels. Indeed, increasing evidence suggests that several forms of post-translational modification (including phosphorylation, acetylation, and ubiquitylation [37-40]) regulate the autophagic flux by influencing the activity, recruitment, and turnover of autophagic components (Table 1 and Figure 1).

Upstream regulators of autophagy such as mTOR and phosphoinositide 3 kinase (PI3K) are post-translationally modified, affecting their activity in response to nutrient availability, ER stress, genotoxic stress, oxidative stress, DNA damage, and infection [10, 27, 37, 41-44].

The number of kinases implicated in the regulation of autophagy continues to increase, and includes eIF2a kinase, TBK1, extracellular signal-regulated kinase (ERK), protein kinase C $(\mathrm{PKC})$, sphingosine kinase, ceramide kinase, $5^{\prime}$ adenosine monophosphate-activated protein kinase (AMPK), IKB kinase (IKK), death-associated protein kinase (DAPK), and Rhoassociated, coiled-coil containing protein kinase 1 (ROCK1) [45-50]. A recent study revealed that loss of ubiquitin protein ligase E3 component n-recognin 4 (UBR4), one of the mammalian $\mathrm{N}$-recognins of the $\mathrm{N}$-end rule pathway, resulted in autophagy [51], implying that $\mathrm{N}$-end rule proteolytic processes also regulate autophagy.

At least two mechanisms exist by which ubiquitylation can regulate autophagy. One is to control the ubiquitylation and stability of upstream autophagy regulators or proteins of the autophagy machinery. The other is to facilitate the recruitment of autophagy adaptors such as p62, neighbor of BRCA1 gene 1(NBR1), histone deacetylase 6 (HDAC6), autophagy receptor BH3-only family protein Nix, the ubiquitin receptor nuclear dot protein $52 \mathrm{kd}$ (Ndp52) and Optineurin, which act as bridging molecules between the ubiquitylated proteins and components of the autophagy machinery [52-56]. However, little is known of the molecular mechanisms by which the ubiquitylation system regulates the autophagy pathways, including which ubiquitin ligases and types of ubiquitin modification are involved. Here, we discuss the emerging role of E3 ubiquitin ligases in the regulation of autophagy (Figure 1). It is worth noting that polyubiquitylated protein aggregates or misfolded proteins can recruit the autophagy machinery for selective autophagy. Therefore, 
the ubiquitin ligases involved in the ubiquitylation of misfolded substrates can also regulate autophagy. Due to the space limitation, this review mainly focuses on the ubiquitin ligases that regulate the autophagy machinery or upstream regulators.

\section{Parkin}

Parkin, a RING-HECT hybrid E3 ligase, plays key functions in mitochondrial homeostasis and often mutates in familial Parkinson's disease. A systematic Parkin-dependent ubiquitylome has revealed multiple functions of Parkin in mitochondrial homeostasis, autophagy and proteasomal degradation [57].

PINK1 (PTEN-induced kinase 1) and Parkin, two proteins linked to familial Parkinson's disease, have been shown to participate in the autophagic degradation of dysfunctional mitochondria (mitophagy) $[58,59]$. Upon phosphorylation by PINK1, Parkin translocates to depolarized mitochondrial membranes and induces the polyubiquitylation (K48-, K63-, or K27-linked) of multiple mitochondrial proteins (e.g., voltage-dependent anion-selective channel protein 1 (VDAC1) and mitofusins Mfn1/2), which leads to the recruitment of the ubiquitin-binding adaptor protein p62, aggregation of the mitochondria, and their clearance by mitophagy [60-65]. The finding that Parkin is mutated in some neurodegenerative diseases, including Parkinson's disease, suggests that aberrant mitophagy may play an important etiological role in these disorders. Along these lines, DJ-1, an adaptor for the PINK1-Parkin complex, has also been implicated in the regulation of Parkin activity. Parkinson's disease is also associated with a mutation in DJ-1 [59] that impairs Parkin activity and reduces its contribution to mitophagy [66]. In addition to mitophagy, Parkin can promote the degradation of misfolded proteins through K63-linked ubiquitylation, which marks these proteins for clearance by autophagy via the adaptor protein HDAC6 [67].

In contrast to its positive role in the autophagic degradation of damaged mitochondria or misfolded proteins, Parkin may also play an inhibitory role in autophagy under normal and starvation conditions by mono-ubiquitylation of the autophagy-inhibiting protein Bcl-2, which increases its steady-state levels and promotes its interaction with Beclin 1 to inhibit autophagy initiation [68]. Parkin is diffusively distributed in the cytoplasm in normal conditions and starvation, but translocates to mitochondria under CCCP treatment that causes mitochondria damage [68]. It seems that Parkin may positively or negatively regulate autophagy, depending on the localization of Parkin and the types of autophagy-inducing stimuli.

\section{Skp, Cullin, F-box containing complex (SCF)}

mTOR is a key negative regulator of autophagy and, accordingly, inhibition of mTOR induces autophagy [42]. The activity of mTORC1 is negatively regulated by an endogenous inhibitor, DEP domain-containing mTOR-interacting protein (DEPTOR) [69, 70], and the abundance of DEPTOR determines the overall activity of the mTOR network. DEPTOR is a substrate of SCF-beta-transducin repeat-containing protein ( $\beta$-TrCP), an E3 ligase that specifically ubiquitylates phosphorylated substrates to promote their degradation [71]. Stimulation of cells with growth factors induces phosphorylation of DEPTOR, leading to its ubiquitylation by $\operatorname{SCF}(\beta-\operatorname{TrCP})$ and subsequent degradation [72-74]. Thus, $\operatorname{SCF}(\beta-\operatorname{TrCP})$ inhibits autophagy by increasing mTOR activity $[73,74]$.

\section{TNF Receptor Associated Factor 6 (TRAF6)}

TRAF6 is a RING (really interesting gene) finger ubiquitin ligase that has been associated with signaling through the IL-1 (interleukin 1) receptor and TLRs (Toll-Like Receptors), primarily in the context of IKK and NF-KB activation [75, 76]. Ligands such as TLR4 (Toll 
Like Receptor 4), IL-1, and IFN-Y (interferon gamma) trigger TRAF6-mediated K63-linked ubiquitylation of their receptors and downstream effectors of the NF-KB pathway.

TRAF6 activation by external stimuli, including TLR4, IL-1, and IFN- $\gamma$, regulates autophagy through ubiquitylation of Beclin 1 [77, 78]. In macrophages, ubiquitylation of Beclin 1 by TRAF6 was shown to be K63-linked and was required for TLR4-dependent autophagy. This effect was antagonized by the deubiquitylating enzyme A20, which limited the extent of Beclin 1 ubiquitylation by TRAF6 and attenuated TLR4-induced autophagy [78]. Insight into how Beclin 1 ubiquitylation might contribute to autophagy comes from earlier studies which have demonstrated that the K63 ubiquitin chains provide a scaffold to "cage" signal transduction components that modify downstream effectors. Thus, the K63linked modification of Beclin 1 may enable more efficient assembly of ATG components required for the autophagy process. Notably, this is the first report of an autophagy-related role for a deubiquitylating (DUB) enzyme. Additional DUBs, including cylindromatosis (CYLD) and the ubiquitin specific protease 4 (USP4), which have been implicated in the control of A20, TRAF6, and the NF-KB pathway [79, 80], may also play a role in autophagy.

Through complex with AMBRA1, TRAF6 also ubiquitylates ULK1. Autophagy induction causes AMBRA dephosphorylation and association with TRAF6, which induces ULK1 ubiquitylation with K63-linked chains, which stabilizes the active ULK1 complex [81].

Finally, TRAF6 has also been implicated in autophagy-mediated clearance of pathogens. CD40 stimulation of macrophages induces vacuole-lysosome fusion through autophagy and results in killing of the obligate intracellular pathogen Toxoplasma gondii. The vacuolelysosome fusion is dependent on synergy between TRAF6 signaling downstream of CD40 and TNF-alpha [82].

\section{RING Finger Protein 5 (RNF5)}

RNF5 is a membrane-bound E3 RING finger ubiquitin ligase localized in ER and mitochondrial membranes that has been implicated in the regulation of substrate stability and localization through K48-linked or K63-linked ubiquitylation, respectively. RNF5 affects numerous cell processes, including cell motility through ubiquitylation of paxillin [83], innate immunity through ubiquitylation of MITA (also known as STING) [84, 85], and ER quality control through ubiquitylation of misfolded proteins, and ubiquitylationdependent relocalization of the proteasome adaptor protein JAMP (JNK associated membrane protein), which contributes to the recruitment of proteasomes at the ER vicinity [86-88]. RNF5 associates with and ubiquitylates membrane-associated ATG4B, a key LC3 protease, which results in its degradation [89]. Notably, RNF5 affects only a fraction of ATG4B that is proximal to the membrane domain where RNF5 resides. Manipulation of RNF5 activity modulates ATG4B levels and concomitantly affects LC3 turnover and formation of autophagosomes (Figure 2), suggesting that membrane nucleation of phagophores and sequestration of autophagosomes is regulated by RNF5. In vivo, RNF5 regulation of autophagy is involved in the innate host defense against bacterial infection. $\mathrm{RNF}^{-/}$mice have been shown to be resistant to group A streptococcus infection, in part due to enhanced autophagy-mediated clearance of invading bacteria by macrophages [89]. Thus, RNF5 control of ATG4B stability constitutes a novel layer in the regulation of autophagy. Interestingly, RNF5 control of ATG4B was mainly observed under conditions when autophagy is not active and/or required (normal growth), thereby limiting the level of autophagy when it was not needed. 
RNF185

RNF185 is a mitochondrial-localized E3 ubiquitin ligase that has been implicated in the regulation of mitophagy. RNF185 is a $21 \mathrm{kDa}$ protein with a RING domain and two transmembrane domains. The Xenopus laevis homologue, XRNF185, associates with and destabilizes paxillin at focal adhesions and promotes cell migration [90], similar to the activity of RNF5, with which RNF185 shares homology [83]. RNF185 mediates K63 ubiquitylation of BNIP1 (BCL2/adenovirus E1B 19kDa interacting protein 1), which enables the recruitment of p62 and LC3 to promote autophagosome formation [91]. However, many aspects of RNF185 biology remain to be explored, including its expression, protein interactions, and physiological role in autophagy.

\section{Nedd4}

Nedd4 (neural precursor cell-expressed developmentally down-regulated 4) is an E3 ligase of the HECT (homologous with E6-associated protein C-terminus) ligase family [92]. A regulatory link between Beclin 1 and Nedd4 was recently demonstrated, in which Nedd4 binds to and mediates K11- and K63-linked ubiquitylation of Beclin 1 [93]. Notably, depletion of Beclin 1 adaptor protein VSP34 resulted in Nedd4-mediated K11 ubiquitylation and degradation of Beclin 1, and inhibition of autophagy. The physiological conditions under which this regulation occurs awaits elucidation.

Human homologue of mouse MDM2 (HDM2)—The cell cycle control protein p53 plays a dual role in the control of autophagy, depending on its subcellular localization. Nuclear p53 can induce autophagy through transcriptional activation of target genes, including autophagy-inducing genes. By contrast, cytoplasmic p53 acts as a repressor of autophagy, although the underlying mechanism is not fully understood [94-96]. HDM2 is an E3 ubiquitin ligase that controls the degradation of p53 [97]. Induction of autophagy stimulates HDM2-dependent ubiquitylation and degradation of cytoplasmic p53, which promotes autophagy. Conversely, autophagy is blocked by inhibition of HDM2 or by expression of a p53 mutant protein that lacks HDM2-binding and ubiquitylation sites [98]. This finding reveals a novel link between autophagy and HDM2 through p53. Although additional E3 ligases can target degradation of cytoplasmic p53 [97], it is not yet known if they play a role in controlling p53-dependent autophagy.

\section{Catalytic activity-independent roles of ubiquitin ligases in autophagy}

Ubiquitin ligases can be largely grouped to 4 main types: those that harbor intrinsic catalytic activity (i.e., HECT domain containing), those that have a RING domain, those in which the RING E3 ligase is part of a multisubunit complex (i.e., SCF), and those harboring U-box domain. Of these four groups, the first group, which harbors intrinsic catalytic activity, has fewer members. Thus, most of the E3 ubiquitin ligases lack intrinsic catalytic activity, relying on the activity of E2 conjugating enzyme to transfer ubiquitin and assemble ubiquitin chains on respective substrates. Given that the regulatory domains of most ligases consist of structural domains implicated in distinct cellular functions, it is expected that ubiquitin ligases may also affect cellular function independent of their ubiquitin-associated functions. The latter may be a result of out-competing a functional cellular protein for binding to a defined target, or assembling a regulatory complex with another catalytically active protein. The following are the initial examples of ubiquitin ligases that have been found to affect autophagy independent of their role in the ubiquitin proteasome pathway.

\section{Tripartite Motif-Containing Protein 13 (TRIM13)}

TRIM13, also known as Rfp2 or Leu5, is a member of the TRIM subfamily of RING family E3 ligases and plays a role in ERAD, ionizing radiation-induced apoptosis, and 
tumorigenesis by targeting MDM2 (mouse double minute 2 homolog) and Akt for ubiquitylation and degradation $[99,100]$. TRIM13 was recently shown to induce autophagy [101]. Under ER stress, TRIM13 initiates autophagy by interacting with p62 and colocalizing with DFCP1 (double FYVE-containing protein 1), an upstream regulator of autophagosome formation. However, the RING domain of TRIM13 is dispensable for this function, indicating that its effect on p62 does not require E3 ligase activity. The mechanisms by which TRIM13 affects p62 and promotes autophagy therefore remain to be elucidated.

Casitas B-lineage Lymphoma ( $\boldsymbol{c}$-Cbl)-The focal adhesion kinase (FAK)-Src tyrosine kinase axis mediates signal transduction by integrins, a large family of cell surface receptors for extracellular matrix proteins [102]. Cbl proteins, a highly conserved family of E3 ligases, regulate diverse signaling pathways including FAK-Src. The interaction between $\mathrm{c}-\mathrm{Cbl}$ and Src induces the ubiquitylation and degradation of both proteins [103]. Active Src was recently shown to be degraded via autophagy in tumor cells, promoting their survival [104, 105]. Upon disruption of FAK signaling, c-Cbl acts as a cargo receptor for Src (as part of the Src-LC3B complex) following the engulfment of active Src into autophagosomes. Notably, $\mathrm{Cbl}$ harbors a LIR domain, enabling interaction with the autophagy components (LC3), which was found to be required for its ability to serve as a cargo receptor for Src in autophagy. Consistent with its cargo-receptor function, $\mathrm{Cbl}$ recruitment of $\mathrm{Src}$ to autophagosomes is independent of its E3 ligase activity. This is another example of a ligaseindependent function for $\mathrm{c}-\mathrm{Cbl}[106,107]$ and emphasizes the role of domains other than the RING finger domain in the increasing number of functions attributed to $\mathrm{c}-\mathrm{Cbl}$.

\section{SMAD specific E3 ubiquitin protein ligase 1 (SMURF1)}

A recent genome-wide siRNA screen identified a ubiquitin ligase-independent function for SMURF1 in selective autophagy [108]. SMURF1 is a HECT E3 ligase belonging to the Nedd4 family and plays multiple physiological functions through targeting a variety of substrates [108]. Its functions in both viral autophagy and mitophagy were found to be tissue-specific and independent of its E3 ligase activity. Although the membrane-targeting domain of SMURF1 was shown to be involved in autophagy, the identity of the autophagic substrate or intermediate remains unknown.

\section{The deubiquitinase SseL}

The role of deubiquitylating enzymes in autophagy remains elusive. The deubiquitylating enzyme A20 can modulate TLR4-induced autophagy by antagonizing the TRAF6-induced ubiquitylation of Beclin 1. [78] Another example that such enzymes can play a regulatory role comes from a study of the Salmonella DUB, SseL [109]. SseL is a $S$. typhimurium virulence protein capable of deubiquitylating the adaptor protein $\mathrm{p} 62$, thereby reducing the recruitment of infection-related protein aggregates to autophagosomes. SseL activity reduces autophagic flux in infected cells and favors bacterial replication, revealing one mechanism by which a bacterial pathogen can counteract the autophagy pathway.

\section{Concluding remarks}

The exponential increase in the number studies of autophagy has revealed its importance for normal homeostasis and the cellular response to stress [7, 10, 15, 17]. Despite this, the intricate molecular mechanisms that control autophagy remain largely elusive. Autophagy is subject to control by post-translational modification (i.e. phosphorylation and ubiquitylation) of components in the autophagic pathways. Most of the ubiquitin ligases involved in autophagy regulation act on upstream autophagy regulators, rather than on autophagy machinery components per se. The exceptions to this are those that regulate the core 
autophagy proteins such as RNF5, which directly modulates the stability of ATG4B; and TRAF6 and Nedd4, which mediate K63-linked ubiquitylation of Beclin 1 and ULK1. It remains to be determined whether more ubiquitin ligases control the stability of autophagy machinery or upstream regulators. In addition, some elicit their effects in a ubiquitylationindependent manner, affecting the recruitment of autophagy proteins or autophagy adaptors. For example, TRIM13 and c-Cbl can recruit the autophagic receptors p62 and LC3, respectively, in a ligase activity-independent manner (Table 1). Further evidence for recruitment of autophagy adaptors by E3 ligases including SMURF1 may arise in future studies. The function of deubiquitylation in autophagy is another area that has received relatively little attention to date. The recent discovery that two deubiquitylating enzymes, USP36 and A20, play a regulatory role in autophagy should increase the interest in deubiquitylation [77, 78, 110]. Future studies aimed at identifying novel E3 ubiquitin ligases and deubiquitylating enzymes will contribute to our understanding of protein ubiquitylation and deubiquitylation under physiological and pathological conditions, and will also aid in the development of novel strategies for therapeutic targeting of autophagy.

\section{Acknowledgments}

This work was supported by NIH grants CA097105 (to ZR), CA154888 (to JQ) and Guangdong Innovative

Research Team Program NO.2009010058 and Sun Yat-Sen University Setup fund (to EK).

\section{References}

1. Mizushima N, Komatsu M. Autophagy: renovation of cells and tissues. Cell. 2011; 147:728-741. [PubMed: 22078875]

2. Van Limbergen J, et al. Autophagy: from basic science to clinical application. Mucosal immunology. 2009; 2:315-330. [PubMed: 19421182]

3. Rabinowitz JD, White E. Autophagy and metabolism. Science. 2010; 330:1344-1348. [PubMed: 21127245]

4. Rubinsztein DC, et al. Autophagy and aging. Cell. 2011; 146:682-695. [PubMed: 21884931]

5. Maiuri MC, et al. Self-eating and self-killing: crosstalk between autophagy and apoptosis. Nature reviews. Molecular cell biology. 2007; 8:741-752.

6. Das G, et al. Regulation and function of autophagy during cell survival and cell death. Cold Spring Harbor perspectives in biology. 2012; 4

7. Kroemer G, et al. Autophagy and the integrated stress response. Molecular cell. 2010; 40:280-293. [PubMed: 20965422]

8. Kuballa P, et al. Autophagy and the immune system. Annual review of immunology. 2012; 30:611646.

9. Levine B, et al. Autophagy in immunity and inflammation. Nature. 2011; 469:323-335. [PubMed: 21248839]

10. Deretic V. Autophagy in infection. Current opinion in cell biology. 2010; 22:252-262. [PubMed: 20116986]

11. Levine B, Kroemer G. Autophagy in the pathogenesis of disease. Cell. 2008; 132:27-42. [PubMed: 18191218]

12. Wong E, Cuervo AM. Autophagy gone awry in neurodegenerative diseases. Nature neuroscience. 2010; 13:805-811.

13. Harris H, Rubinsztein DC. Control of autophagy as a therapy for neurodegenerative disease. Nature reviews. Neurology. 2012; 8:108-117.

14. Kimmelman AC. The dynamic nature of autophagy in cancer. Genes \& development. 2011; 25:1999-2010. [PubMed: 21979913]

15. Janku F, et al. Autophagy as a target for anticancer therapy. Nature reviews. Clinical oncology. 2011; 8:528-539. 
16. Rubinsztein DC, et al. Potential therapeutic applications of autophagy. Nature reviews. Drug discovery. 2007; 6:304-312.

17. Rubinsztein DC, et al. Autophagy modulation as a potential therapeutic target for diverse diseases. Nature reviews. Drug discovery. 2012; 11:709-730.

18. Hershko A, Ciechanover A. The ubiquitin system. Annual review of biochemistry. 1998; 67:425479.

19. Varshavsky A. The ubiquitin system, an immense realm. Annual review of biochemistry. 2012; 81:167-176.

20. Pickart CM. Mechanisms underlying ubiquitination. Annual review of biochemistry. 2001; 70:503-533.

21. Deshaies RJ, Joazeiro CA. RING domain E3 ubiquitin ligases. Annual review of biochemistry. 2009; 78:399-434.

22. Komander D, Rape M. The ubiquitin code. Annual review of biochemistry. 2012; 81:203-229.

23. Xu G, Jaffrey SR. The new landscape of protein ubiquitination. Nature biotechnology. 2011; 29:1098-1100.

24. Xu P, et al. Quantitative proteomics reveals the function of unconventional ubiquitin chains in proteasomal degradation. Cell. 2009; 137:133-145. [PubMed: 19345192]

25. Kravtsova-Ivantsiv Y, Ciechanover A. Non-canonical ubiquitin-based signals for proteasomal degradation. Journal of cell science. 2012; 125:539-548. [PubMed: 22389393]

26. Kundu M, Thompson CB. Autophagy: basic principles and relevance to disease. Annual review of pathology. 2008; 3:427-455.

27. Weidberg H, et al. Biogenesis and cargo selectivity of autophagosomes. Annual review of biochemistry. 2011; 80:125-156.

28. Nakatogawa H, et al. Dynamics and diversity in autophagy mechanisms: lessons from yeast. Nature reviews. Molecular cell biology. 2009; 10:458-467.

29. Romanov J, et al. Mechanism and functions of membrane binding by the Atg5-Atg12/Atg16 complex during autophagosome formation. The EMBO journal. 2012

30. Hanada T, et al. The Atg12-Atg5 conjugate has a novel E3-like activity for protein lipidation in autophagy. The Journal of biological chemistry. 2007; 282:37298-37302. [PubMed: 17986448]

31. Geng J, Klionsky DJ. The Atg8 and Atg12 ubiquitin-like conjugation systems in macroautophagy. 'Protein modifications: beyond the usual suspects' review series. EMBO reports. 2008; 9:859-864. [PubMed: 18704115]

32. Popovic D, et al. Rab GTPase-activating proteins in autophagy: regulation of endocytic and autophagy pathways by direct binding to human ATG8 modifiers. Molecular and cellular biology. 2012; 32:1733-1744. [PubMed: 22354992]

33. Tanida I, et al. HsAtg4B/HsApg4B/autophagin-1 cleaves the carboxyl termini of three human Atg8 homologues and delipidates microtubule-associated protein light chain 3- and GABAA receptorassociated protein-phospholipid conjugates. The Journal of biological chemistry. 2004; 279:36268-36276. [PubMed: 15187094]

34. Yu ZQ, et al. Dual roles of Atg8-PE deconjugation by Atg4 in autophagy. Autophagy. 2012; 8:883-892. [PubMed: 22652539]

35. Nair U, et al. A role for Atg8-PE deconjugation in autophagosome biogenesis. Autophagy. 2012; 8:780-793. [PubMed: 22622160]

36. Nakatogawa $\mathrm{H}$, et al. Atg4 recycles inappropriately lipidated Atg8 to promote autophagosome biogenesis. Autophagy. 2012; 8:177-186. [PubMed: 22240591]

37. Alers $\mathrm{S}$, et al. Role of AMPK-mTOR-Ulk1/2 in the regulation of autophagy: cross talk, shortcuts, and feedbacks. Molecular and cellular biology. 2012; 32:2-11. [PubMed: 22025673]

38. Liang C. Negative regulation of autophagy. Cell death and differentiation. 2010; 17:1807-1815. [PubMed: 20865012]

39. McEwan DG, Dikic I. The Three Musketeers of Autophagy: phosphorylation, ubiquitylation and acetylation. Trends in cell biology. 2011; 21:195-201. [PubMed: 21277210]

40. Hamai A, Codogno P. New targets for acetylation in autophagy. Science signaling. 2012; 5:pe29. [PubMed: 22763338] 
41. He C, Klionsky DJ. Regulation mechanisms and signaling pathways of autophagy. Annual review of genetics. 2009; 43:67-93.

42. Neufeld TP. TOR-dependent control of autophagy: biting the hand that feeds. Current opinion in cell biology. 2010; 22:157-168. [PubMed: 20006481]

43. Deretic V, Levine B. Autophagy, immunity, and microbial adaptations. Cell host \& microbe. 2009; 5:527-549. [PubMed: 19527881]

44. Kamada Y, et al. Autophagy in yeast: a TOR-mediated response to nutrient starvation. Current topics in microbiology and immunology. 2004; 279:73-84. [PubMed: 14560952]

45. Mleczak A, et al. Regulation of autophagosome formation by Rho kinase. Cellular signalling. 2012

46. Mack HI, et al. AMPK-dependent phosphorylation of ULK1 regulates ATG9 localization. Autophagy. 2012; 8

47. Liu W, Phang JM. Proline dehydrogenase (oxidase), a mitochondrial tumor suppressor, and autophagy under the hypoxia microenvironment. Autophagy. 2012; 8:1407-1409. [PubMed: 22885468]

48. Lepine S, et al. Autophagy induced by deficiency of sphingosine-1-phosphate phosphohydrolase 1 is switched to apoptosis by calpain-mediated autophagy-related gene 5 (Atg5) cleavage. The Journal of biological chemistry. 2011; 286:44380-44390. [PubMed: 22052905]

49. Bialik S, Kimchi A. Lethal weapons: DAP-kinase, autophagy and cell death: DAP-kinase regulates autophagy. Current opinion in cell biology. 2010; 22:199-205. [PubMed: 20005690]

50. Sakaki K, Kaufman RJ. Regulation of ER stress-induced macroautophagy by protein kinase C. Autophagy. 2008; 4:841-843. [PubMed: 18670192]

51. Tasaki T, et al. UBR box N-recognin-4 (UBR4), an N-recognin of the N-end rule pathway, and its role in yolk sac vascular development and autophagy. Proceedings of the National Academy of Sciences of the United States of America. 2013; 110:3800-3805. [PubMed: 23431188]

52. von Muhlinen N, et al. NDP52, a novel autophagy receptor for ubiquitin-decorated cytosolic bacteria. Autophagy. 2010; 6:288-289. [PubMed: 20104023]

53. Korac J, et al. Ubiquitin-independent function of optineurin in autophagic clearance of protein aggregates. Journal of cell science. 2013; 126:580-592. [PubMed: 23178947]

54. Kanki T. Nix, a receptor protein for mitophagy in mammals. Autophagy. 2010; 6:433-435. [PubMed: 20200478]

55. Lee JY, et al. HDAC6 controls autophagosome maturation essential for ubiquitin-selective qualitycontrol autophagy. The EMBO journal. 2010; 29:969-980. [PubMed: 20075865]

56. Moscat J, Diaz-Meco MT. Feedback on fat: p62-mTORC1-autophagy connections. Cell. 2011; 147:724-727. [PubMed: 22078874]

57. Sarraf SA, et al. Landscape of the PARKIN-dependent ubiquitylome in response to mitochondrial depolarization. Nature. 2013; 496:372-376. [PubMed: 23503661]

58. Narendra D, et al. Parkin is recruited selectively to impaired mitochondria and promotes their autophagy. The Journal of cell biology. 2008; 183:795-803. [PubMed: 19029340]

59. Xiong H, et al. Parkin, PINK1, and DJ-1 form a ubiquitin E3 ligase complex promoting unfolded protein degradation. The Journal of clinical investigation. 2009; 119:650-660. [PubMed: 19229105]

60. Kim Y, et al. PINK1 controls mitochondrial localization of Parkin through direct phosphorylation. Biochemical and biophysical research communications. 2008; 377:975-980. [PubMed: 18957282]

61. Lee JY, et al. Disease-causing mutations in parkin impair mitochondrial ubiquitination, aggregation, and HDAC6-dependent mitophagy. The Journal of cell biology. 2010; 189:671-679. [PubMed: 20457763]

62. Geisler S, et al. PINK1/Parkin-mediated mitophagy is dependent on VDAC1 and p62/SQSTM1. Nature cell biology. 2010; 12:119-131.

63. Chan NC, et al. Broad activation of the ubiquitin-proteasome system by Parkin is critical for mitophagy. Human molecular genetics. 2011; 20:1726-1737. [PubMed: 21296869]

64. Tanaka A, et al. Proteasome and p97 mediate mitophagy and degradation of mitofusins induced by Parkin. The Journal of cell biology. 2010; 191:1367-1380. [PubMed: 21173115] 
65. Gegg ME, et al. Mitofusin 1 and mitofusin 2 are ubiquitinated in a PINK1/parkin-dependent manner upon induction of mitophagy. Human molecular genetics. 2010; 19:4861-4870. [PubMed: 20871098]

66. Heo JY, et al. DJ-1 null dopaminergic neuronal cells exhibit defects in mitochondrial function and structure: involvement of mitochondrial complex I assembly. PloS one. 2012; 7:e32629. [PubMed: 22403686]

67. Olzmann JA, Chin LS. Parkin-mediated K63-linked polyubiquitination: a signal for targeting misfolded proteins to the aggresome-autophagy pathway. Autophagy. 2008; 4:85-87. [PubMed: 17957134]

68. Chen D, et al. Parkin mono-ubiquitinates Bcl-2 and regulates autophagy. The Journal of biological chemistry. 2010; 285:38214-38223. [PubMed: 20889974]

69. Peterson TR, et al. DEPTOR is an mTOR inhibitor frequently overexpressed in multiple myeloma cells and required for their survival. Cell. 2009; 137:873-886. [PubMed: 19446321]

70. Liu M, et al. Resveratrol inhibits mTOR signaling by promoting the interaction between mTOR and DEPTOR. The Journal of biological chemistry. 2010; 285:36387-36394. [PubMed: 20851890]

71. Frescas D, Pagano M. Deregulated proteolysis by the F-box proteins SKP2 and beta-TrCP: tipping the scales of cancer. Nature reviews. Cancer. 2008; 8:438-449.

72. Wang Z, et al. DEPTOR ubiquitination and destruction by SCF(beta-TrCP). American journal of physiology. Endocrinology and metabolism. 2012; 303:E163-169. [PubMed: 22454292]

73. Zhao Y, et al. DEPTOR, an mTOR inhibitor, is a physiological substrate of SCF(betaTrCP) E3 ubiquitin ligase and regulates survival and autophagy. Molecular cell. 2011; 44:304-316. [PubMed: 22017876]

74. Gao D, et al. mTOR drives its own activation via $\mathrm{SCF}$ (betaTrCP)-dependent degradation of the mTOR inhibitor DEPTOR. Molecular cell. 2011; 44:290-303. [PubMed: 22017875]

75. Wang Y, et al. Association of beta-arrestin and TRAF6 negatively regulates Toll-like receptorinterleukin 1 receptor signaling. Nature immunology. 2006; 7:139-147. [PubMed: 16378096]

76. Deng L, et al. Activation of the IkappaB kinase complex by TRAF6 requires a dimeric ubiquitinconjugating enzyme complex and a unique polyubiquitin chain. Cell. 2000; 103:351-361. [PubMed: 11057907]

77. Shi CS, Kehrl JH. Traf6 and A20 differentially regulate TLR4-induced autophagy by affecting the ubiquitination of Beclin 1. Autophagy. 2010; 6:986-987. [PubMed: 20798608]

78. Shi CS, Kehrl JH. TRAF6 and A20 regulate lysine 63-linked ubiquitination of Beclin-1 to control TLR4-induced autophagy. Science signaling. 2010; 3:ra42. [PubMed: 20501938]

79. Yoshida $\mathrm{H}$, et al. The tumor suppressor cylindromatosis (CYLD) acts as a negative regulator for toll-like receptor 2 signaling via negative cross-talk with TRAF6 AND TRAF7. The Journal of biological chemistry. 2005; 280:41111-41121. [PubMed: 16230348]

80. Xiao N, et al. Ubiquitin-specific protease 4 (USP4) targets TRAF2 and TRAF6 for deubiquitination and inhibits TNFalpha-induced cancer cell migration. The Biochemical journal. 2012; 441:979-986. [PubMed: 22029577]

81. Nazio F, et al. mTOR inhibits autophagy by controlling ULK1 ubiquitylation, self-association and function through AMBRA1 and TRAF6. Nature cell biology. 2013; 15:406-416.

82. Tokito F, et al. Epidermal growth factor (EGF) decreased endothelin-2 (ET-2) production in human renal adenocarcinoma cells. FEBS letters. 1991; 295:17-21. [PubMed: 1722462]

83. Didier C, et al. RNF5, a RING finger protein that regulates cell motility by targeting paxillin ubiquitination and altered localization. Molecular and cellular biology. 2003; 23:5331-5345. [PubMed: 12861019]

84. Zhong B, et al. The ubiquitin ligase RNF5 regulates antiviral responses by mediating degradation of the adaptor protein MITA. Immunity. 2009; 30:397-407. [PubMed: 19285439]

85. Zhong B, et al. The E3 ubiquitin ligase RNF5 targets virus-induced signaling adaptor for ubiquitination and degradation. J Immunol. 2010; 184:6249-6255. [PubMed: 20483786]

86. Younger JM, et al. Sequential quality-control checkpoints triage misfolded cystic fibrosis transmembrane conductance regulator. Cell. 2006; 126:571-582. [PubMed: 16901789] 
87. Morito D, et al. Gp78 cooperates with RMA1 in endoplasmic reticulum-associated degradation of CFTRDeltaF508. Molecular biology of the cell. 2008; 19:1328-1336. [PubMed: 18216283]

88. Tcherpakov M, et al. Regulation of endoplasmic reticulum-associated degradation by RNF5dependent ubiquitination of JNK-associated membrane protein (JAMP). The Journal of biological chemistry. 2009; 284:12099-12109. [PubMed: 19269966]

89. Kuang E, et al. Regulation of ATG4B Stability by RNF5 Limits Basal Levels of Autophagy and Influences Susceptibility to Bacterial Infection. PLoS genetics. 2012; 8:e1003007. [PubMed: 23093945]

90. Iioka $\mathrm{H}$, et al. Wnt signalling regulates paxillin ubiquitination essential for mesodermal cell motility. Nature cell biology. 2007; 9:813-821.

91. Tang F, et al. RNF185, a novel mitochondrial ubiquitin E3 ligase, regulates autophagy through interaction with BNIP1. PloS one. 2011; 6:e24367. [PubMed: 21931693]

92. Rotin D, Kumar S. Physiological functions of the HECT family of ubiquitin ligases. Nature reviews. Molecular cell biology. 2009; 10:398-409.

93. Platta HW, et al. Nedd4-dependent lysine-11-linked polyubiquitination of the tumour suppressor Beclin 1. The Biochemical journal. 2012; 441:399-406. [PubMed: 21936852]

94. Tasdemir E, et al. A dual role of p53 in the control of autophagy. Autophagy. 2008; 4:810-814. [PubMed: 18604159]

95. Maiuri MC, et al. Autophagy regulation by p53. Current opinion in cell biology. 2010; 22:181185. [PubMed: 20044243]

96. Galluzzi L, et al. Defective autophagy control by the p53 rheostat in cancer. Cell Cycle. 2010; 9:250-255. [PubMed: 20023384]

97. Dai C, Gu W. p53 post-translational modification: deregulated in tumorigenesis. Trends in molecular medicine. 2010; 16:528-536. [PubMed: 20932800]

98. Tasdemir E, et al. Regulation of autophagy by cytoplasmic p53. Nature cell biology. 2008; 10:676687.

99. Lerner M, et al. The RBCC gene RFP2 (Leu5) encodes a novel transmembrane E3 ubiquitin ligase involved in ERAD. Molecular biology of the cell. 2007; 18:1670-1682. [PubMed: 17314412]

100. Joo HM, et al. Ret finger protein 2 enhances ionizing radiation-induced apoptosis via degradation of AKT and MDM2. European journal of cell biology. 2011; 90:420-431. [PubMed: 21333377]

101. Tomar D, et al. TRIM13 regulates ER stress induced autophagy and clonogenic ability of the cells. Biochimica et biophysica acta. 2012; 1823:316-326. [PubMed: 22178386]

102. Mitra SK, Schlaepfer DD. Integrin-regulated FAK-Src signaling in normal and cancer cells. Current opinion in cell biology. 2006; 18:516-523. [PubMed: 16919435]

103. Ryan PE, et al. Regulating the regulator: negative regulation of $\mathrm{Cbl}$ ubiquitin ligases. Trends in biochemical sciences. 2006; 31:79-88. [PubMed: 16406635]

104. Cecconi F. c-Cbl targets active Src for autophagy. Nature cell biology. 2012; 14:48-49.

105. Sandilands E, et al. Autophagic targeting of Src promotes cancer cell survival following reduced FAK signalling. Nature cell biology. 2012; 14:51-60.

106. Zhou L, Yang H. The von Hippel-Lindau tumor suppressor protein promotes c-Cbl-independent poly-ubiquitylation and degradation of the activated EGFR. PloS one. 2011; 6:e23936. [PubMed: 21949687]

107. Thien CB, et al. RING finger mutations that abolish c-Cbl-directed polyubiquitination and downregulation of the EGF receptor are insufficient for cell transformation. Molecular cell. 2001; 7:355-365. [PubMed: 11239464]

108. Orvedahl A, et al. Image-based genome-wide siRNA screen identifies selective autophagy factors. Nature. 2011; 480:113-117. [PubMed: 22020285]

109. Thomas M, et al. The DUB-ious lack of ALIS in Salmonella infection: A Salmonella deubiquitinase regulates the autophagy of protein aggregates. Autophagy. 2012; 8

110. Taillebourg E, et al. The deubiquitinating enzyme USP36 controls selective autophagy activation by ubiquitinated proteins. Autophagy. 2012; 8:767-779. [PubMed: 22622177] 
111. Gegg ME, Schapira AH. PINK1-parkin-dependent mitophagy involves ubiquitination of mitofusins 1 and 2: Implications for Parkinson disease pathogenesis. Autophagy. 2011; 7:243245. [PubMed: 21139416] 


\section{Outstanding Questions Box}

- Are select ubiquitin ligases dedicated for control of autophagy?

- Which components of the autophagy pathways are subjective to the control by the preferred form of ubiquitylation (topology)?

- Which post-translational modifications are required to regulate the function of ubiquitin ligase in autophagy?

- What are the catalytic-independent functions of ubiquitin ligases in autophagy?

- Would ubiquitin ligases involved in processes that limit misfolded proteins (i.e. ERAD), also contribute to control of autophagy? 


\section{Box 1. Roles and regulation of Ubiquitin E3 ligases in autophagy}

Ubiquitylation plays central roles in controlling the stabilization, localization, activation and binding partners of proteins. A variety of ubiquitin E3 ligases have been demonstrated to regulate autophagy. At least two distinct mechanisms have been revealed by which ubiquitylation regulates autophagy: catalytic activity-dependent E3 ligases regulate degradation or stabilization of autophagic machinery and activity-independent E3 ligases regulate recruitment of autophagy adaptors. However, the rules and models of selective substrates and adaptors have not been interpreted. Further studies identifying novel E3 ligases, their substrates, the mechanism of recruitment of adaptors by E3 ligases involved in autophagy, as well as systematic analysis of the ubiquitylome of the E3 ligases involved in autophagy, will provide new insights to autophagic regulation. 


\section{Highlights}

Sequential conjugation of ATGs mimics the ubiquitin conjugation process.

Most ubiquitin ligases involved in autophagy act on upstream regulatory components.

RNF5, TRAF6, Nedd4 exemplify ubiquitin ligases that affect the key autophagy components ATG4B and Beclin 1. 


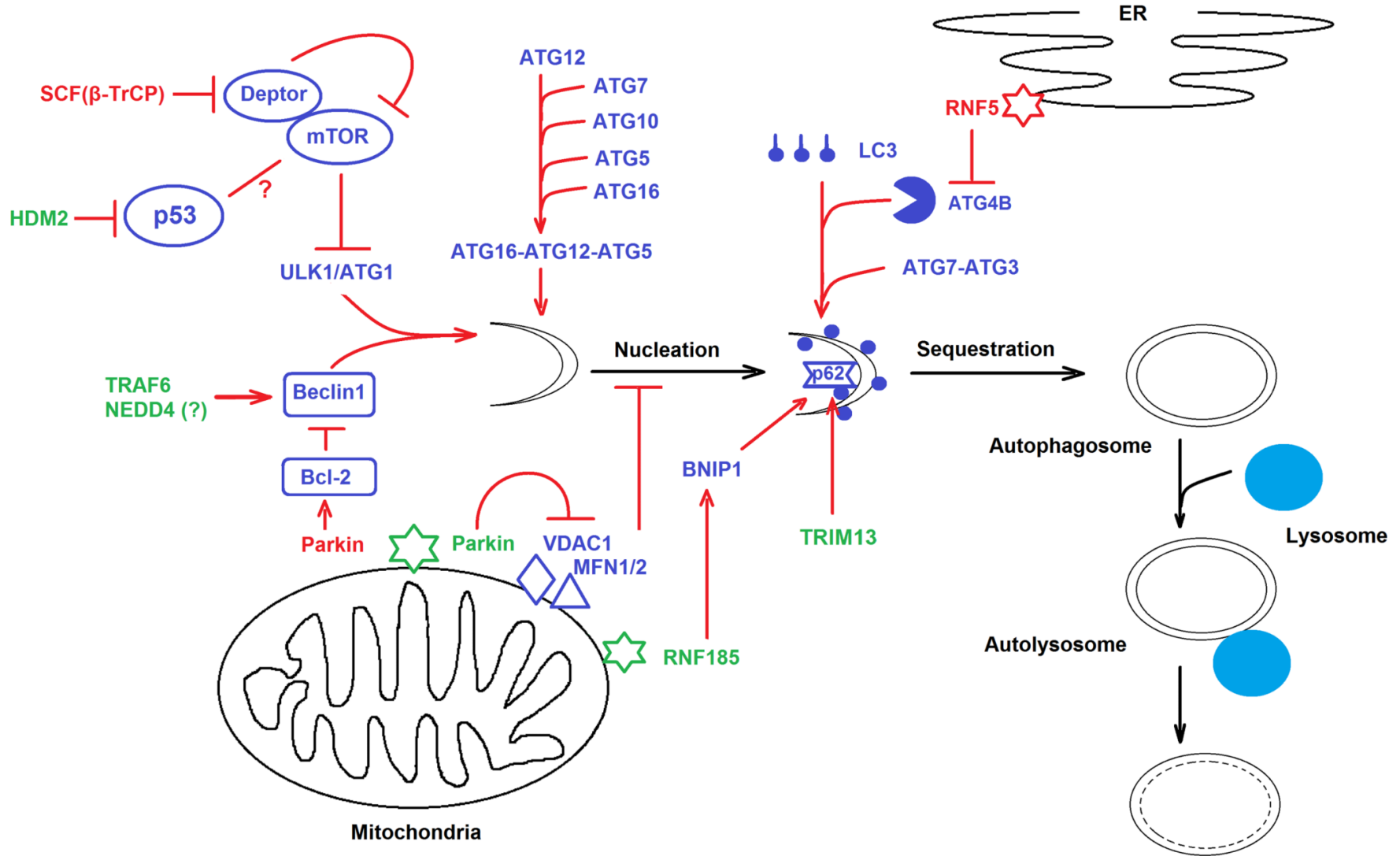

Figure 1. E3 ligases and their targets in autophagy

The figure points to currently known E3 ubiquitin ligases implicated in the control of autophagy. E3 ligases thought to play negative regulatory roles [RNF5, SCF $(\beta-\operatorname{TrCP})$, and Parkin] are shown in red and those thought to play positive roles (HDM2, TRAF6, RNF185, Parkin, and TRIM13) are shown in green. The respective cellular pathways or organelles associated with the activity of these ubiquitin ligases are shown. The respective sections in the text provide details per the known role and underlying mechanisms. '?' indicate further studies are required to substantiate the proposed links. RNF5 - RING Finger Protein 5; HDM2 - human homologue of mouse MDM2; TRAF6 - TNF Receptor Associated Factor 6; RNF185- RING Finger protein 185;SCF-(Skp, Cullin, F-box containing complex); $\beta$-TrCP beta-transducin repeat-containing protein). 
A

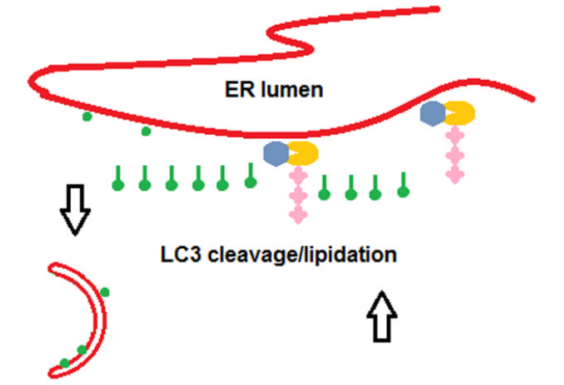

Phagophore<smiles>c1ccccc1</smiles>

Autophagosome

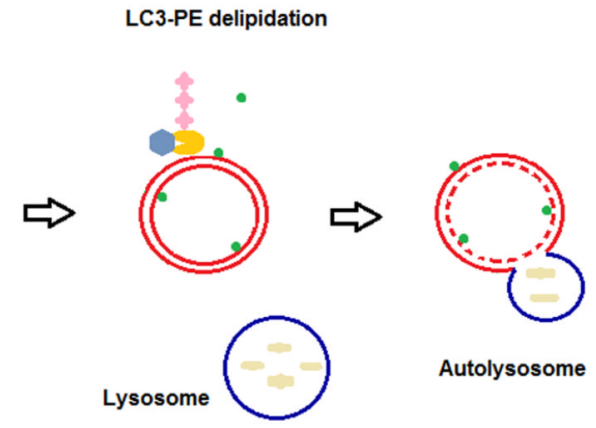

\ Full length LC3

- LC3-PE

3 ATG4B

- RNF5

Ubiquitin

Autolysosome
B

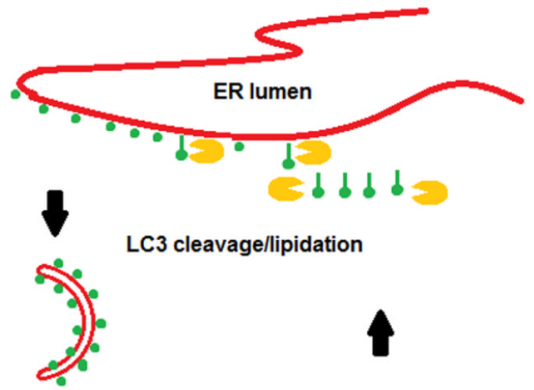

Phagophor

LC3-PE delipidation
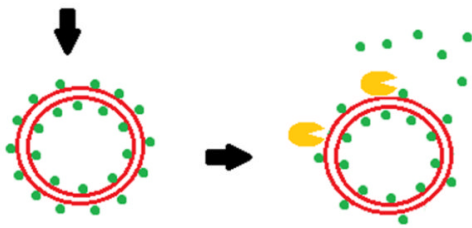

Autophagosome
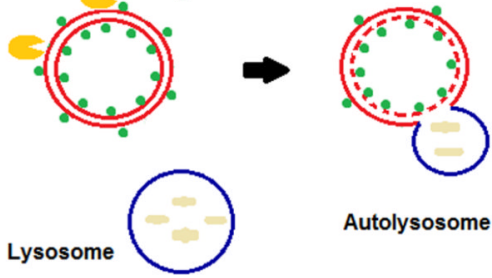

Figure 2. Regulation of autophagy by RNF5

(A) In RNF5 ${ }^{+/+}$cells, RNF5 mediates ubiquitylation and degradation of ATG4B in the proximity of membranes, given that RNF5 is anchored in membranes [represented by pink diamond shape (ubiquitin) and blue hexagon (RNF5) bound to yellow marked ATG4B and affecting LC3 processing (green)], which reduces LC3 processing. As a result, RNF5 limits LC3 turnover and the initiation and formation of autophagosomes. Empty arrows represent decreased flux of phagophore-autophagosome formation, as well as recycling (delipidation). (B) In RNF5 ${ }^{-1-}$ cells, ATG4B is recruited to the membrane compartment where it cleaves full-length LC3 and deconjugates or recycles LC3 on the outer membrane of the autophagosome. Accumulated levels of LC3-I are converted to LC3-II, facilitating the initiation and formation of autophagosomes. Filled arrows point to the increased flux of phagophore-autophagosome formation and recycling. 
Table 1

E3 ligases and target proteins involved in autophagy

\begin{tabular}{lllc}
\hline E3 ligase & Target & Impact on autophagy & References \\
\hline \multicolumn{2}{l}{ Catalytic-dependent function } & & \\
Parkin & Mfn1 and Mfn2 & Induces mitophagy & {$[64,111]$} \\
& VDAC1 & Induces mitophagy & {$[62]$} \\
& Bcl-2 & Inhibits autophagy by stabilizing Bcl-2 & {$[68]$} \\
SCF( $\beta$-TrCP) & DEPTOR & Inhibits autophagy through mTOR signaling & {$[73]$} \\
HDM2 & p53 & Activates autophagy through cytoplasmic p53 & {$[98]$} \\
RNF185 & BNIP1 & Induces selective mitophagy & {$[91]$} \\
Nedd4 & Beclin 1 & $?$ & {$[93]$} \\
RNF5 & ATG4B & Limits LC3 turnover and autophagy & {$[89]$} \\
TRAF6 & Beclin 1 & Mediates TLR4-induced autophagy & {$[77,78]$} \\
& ULK1 & Mediates autophagy by stabilizing ULK1 & {$[81]$} \\
Catalytic-independent function & & {$[104,105]$} \\
TRIM13 & p62/SQSTM1 & Induces autophagy & {$[108]$} \\
c-Cbl & Src & Regulates Src-LC3B interaction & \\
SMURF1 & $?$ & Mediates both viral autophagy and mitophagy & \\
\hline
\end{tabular}

